\title{
RESEARCH
}

Open Access

\section{Dietary behaviors throughout childhood are associated with adiposity and estimated insulin resistance in early adolescence: a longitudinal study}

Véronique Gingras $^{1 *}$ D, Sheryl L. Rifas-Shiman ${ }^{1}$, Elsie M. Taveras ${ }^{2}$, Emily Oken ${ }^{1,3}$ and Marie-France Hivert ${ }^{1,4}$

\begin{abstract}
Background: Despite the growing prevalence of excess weight and prediabetes in children, the contributing role of dietary behaviors throughout childhood remains poorly understood. We examined longitudinal associations of dietary behaviors throughout childhood with adiposity and estimated insulin resistance (HOMA-IR) in adolescence.

Methods: Among 995 children from Project Viva, a pre-birth cohort, we examined associations of child dietary behaviors (frequency of eating breakfast, fast food, family dinner, and eating meals while watching television) reported annually throughout childhood (from ages 4 to 11 years) with body mass index $z$-score (BMI-z; $n=991$ ), waist circumference (WC; $n=995)$, DXA overall and central adiposity measurements $(n=721)$, and HOMA-IR $(n=579)$ in early adolescence (13.2 \pm 0.9 years old). We used mixed effects models adjusted for potential confounders.

Results: Eating breakfast daily throughout childhood was associated with lower BMI-z and DXA-measured overall and central adiposity in boys and girls (e.g. for whole-body fat \%: $\beta-1.43 \%$ [95\% Cl: $-2.42,-0.45]$ and $-1.47 \%[-2.25,-0.68]$ ), and with lower HOMA-IR in boys (\% difference $-15.6 \%[-22.7,-7.9]$ ). Daily family dinner and eating fast food less than once per week throughout childhood were both associated with lower BMI- $z$ and adiposity in girls (for BMI-z: $\beta-0.17$ units $[-0.24,-0.11]$ and $\beta-0.09$ units $[-0.17,-0.02]$ ) and lower insulin resistance in boys (\% difference $-7.3 \%[-12.4,-1.8]$ and $-7.6 \%[-13.2,-1.7])$. Finally, eating meals while watching television $<1$ /week throughout childhood was associated with lower adolescent adiposity (e.g. WC: $-1.55 \mathrm{~cm}[-2.39,-0.71]$ ) and HOMA-IR (\% difference: $-10.7 \%[-15.8,-5.2]$ ) in boys.

Conclusion: Healthful dietary behaviors throughout childhood are associated with less adiposity and lower estimated insulin resistance in early adolescence.
\end{abstract}

Trial registration: NCT02820402

Keywords: Dietary behaviors, Childhood, Adolescence, Breakfast, Family dinner, Television, Fast-food, Adiposity, Insulin resistance

\footnotetext{
* Correspondence: veronique_gingras@harvardpilgrim.org

'Division of Chronic Disease Research Across the Lifecourse, Department of

Population Medicine, Harvard Medical School and Harvard Pilgrim Health

Care Institute, Landmark Center, 401 Park Drive Suite 401, Boston, MA 02215,

USA

Full list of author information is available at the end of the article
}

(c) The Author(s). 2018 Open Access This article is distributed under the terms of the Creative Commons Attribution 4.0 International License (http://creativecommons.org/licenses/by/4.0/), which permits unrestricted use, distribution, and reproduction in any medium, provided you give appropriate credit to the original author(s) and the source, provide a link to the Creative Commons license, and indicate if changes were made. The Creative Commons Public Domain Dedication waiver (http://creativecommons.org/publicdomain/zero/1.0/) applies to the data made available in this article, unless otherwise stated. 


\section{Background}

Over the past decades, childhood obesity has become a global epidemic [1], and it is associated with an early appearance of complications that have been traditionally recognized as adult diseases. For example, type 2 diabetes has been increasingly reported in children and adolescents $[2,3]$ and this increasing prevalence of type 2 diabetes in children parallels the increasing prevalence of obesity and overweight. Pediatric obesity is multifactorial and can be caused by a vast array of genetic, behavioral and environmental factors $[4,5]$.

Several lifestyle components including unhealthy dietary behaviors have been associated with the rising prevalence of childhood obesity and related cardiometabolic disorders [6]. However, previous observational reports often did not account for potential confounders and did not have longitudinal assessment over multiple time points during childhood to investigate long-term effects of lifestyle behaviors. In addition, little is known about the contributing role of dietary behaviors to insulin resistance and type 2 diabetes in children, and whether these associations, if present, are independent of adiposity.

The aim of this study was to examine longitudinal associations of dietary behaviors including frequency of eating breakfast, family dinner, eating fast food, and eating meals while watching television, throughout childhood with obesity, body composition, and estimated insulin resistance in early adolescence. A better understanding of the long-term effects of these dietary behaviors on risk of childhood obesity and insulin resistance could help improve the focus of family-based interventions in children.

\section{Methods}

\section{Subjects}

We studied participants from Project Viva, a longitudinal pre-birth cohort from eastern Massachusetts, United States, established to examine associations of pre- and postnatal factors with maternal and child health. Between 1999 and 2003, we recruited women who understood English, were at $<22$ weeks of pregnancy and with a singleton pregnancy during their first prenatal visit at one of the participating clinics from Atrius Harvard Vanguard Medical Associates [7]. We previously published a full cohort description, including recruitment methods [7] and we registered Project Viva as NCT02820402. All study instruments and protocols are available online, at https://www.hms.harvard.edu/ viva/protocol-policies.html.

Women who agreed to participate completed in-person visits during early pregnancy, mid-pregnancy, and at delivery. Mothers and children then completed in-person visits in infancy (6 months), early (mean age, 3.2 years old), and mid-childhood (mean age, 7.9 years old) as well as early adolescence (mean age, 13.2 years).
Every year beginning at 1 year postpartum, we mailed questionnaires to mothers, and we sent questionnaires to children as well starting at age nine years. From the original 2128 live-born singletons, 1684 remained eligible (did not disenroll) for the early teen visit and 1038 completed the in-person study visit. Of 1038, we excluded 7 with missing covariate data and 36 with no data for dietary behaviors throughout childhood, thus the final analysis sample included 995 participants (Additional file 1: Figure S1).

The 995 included compared to the 1133 excluded participants from the initial cohort were similar in terms of maternal pre-pregnancy BMI (mean 24.8 vs. $25.0 \mathrm{~kg} / \mathrm{m}^{2}$ ), marital status (92 vs. $91 \%$ married or living together), parity (47\% vs. $48 \%$ nulliparous), and child race/ethnicity (65 vs. $63 \%$ white). Women included were more likely to have completed a college degree (71 vs. 59\%) and had higher annual household income (64 vs. 58\% reported > US $\$ 70,000 /$ year).

The institutional review board of Harvard Pilgrim Health Care approved this study and all procedures were in accordance with the ethical standards for human experimentation established by the Declaration of Helsinki. All mothers provided written informed consent at enrollment and at each postpartum in-person visit, and children provided verbal assent at the early teen visit.

\section{Measurements \\ Exposures}

We obtained data on dietary behaviors from self-administered questionnaires. We sent yearly questionnaires (by mail and/or email) to mothers who were still enrolled in the cohort, except for at the mid-childhood when the questionnaires were self-administered during in-person visits at home or at the research facility. Starting at age nine years, participating children themselves also completed yearly questionnaires. Thus, for this study, the exposures are based on maternal reports from ages four to eight years and child reports from ages nine to eleven years. Although questionnaires were sent starting at one-year postpartum, the dietary behaviors of interest in this analysis (exposures) were included in the questionnaires beginning at age four. The number of collected questionnaires at each visit from age four to age eleven ranged from 584 to 916 (59 to $92 \%$ of included sample per year; Additional file 1: Figure S2).

We examined four dietary behaviors in this study: eating breakfast, eating dinner together with family, eating fast food, and eating meals while watching television. We questioned participants on the average frequency of these behaviors over the previous month. For example, for family dinner, the question was: In the past month, on average, how often does your child eat supper or dinner together with family members ("family dinner")? 
Answers ranged on a five-level scale, from "never/less than once per week" to either "every day" or "five times per week or more", depending on the question. For analysis purposes, we categorized eating breakfast and eating dinner together with family as "always/daily" versus " $\leq$ six times per week". We categorized fast food consumption and eating meals while watching television as "less than once per week (between zero and three times per month)" versus " $\geq$ once per week". Categorization was based upon available choices of answers in questionnaires through time, and so that for each behavior we were comparing the hypothesized beneficial direction of exposure compared with the less beneficial exposure. All four behaviors were reported annually from age four to eleven except for breakfast eating, which we did not assess at age eleven.

\section{Outcomes}

Outcomes included children's adiposity measurements and estimated insulin resistance at early adolescence (mean \pm SD: $13.2 \pm 0.9$ years old). Research assistants performing all measurements completed regular training and used standardized techniques. During the in-person visit, we measured weight (TBF-300A scale, Tanita, Arlington Heights, IL) and standing height (calibrated stadiometer, Shorr Productions, Olney, MD). We calculated age and sex-specific BMI z-scores (BMI-z) using U.S. national reference data [8]. We measured waist circumference just above the iliac crest in standing participants with waist exposed using a Gulick II measuring tape (Performance Health, Warrenville, IL) [9]. We measured whole-body fat percentage with bio-impedance (BI) analysis using the TBF-300A scale. We also performed dual-energy X-ray absorptiometry (DXA) scans (Hologic model Discovery A, Bedford, MA) for participants who completed the in-person visit at the research facility $(n=721)$. A quality control check of the DXA scan (inspection and daily calibration with a standard phantom from the manufacturer to assess for machine drift), as per manufacturer's instructions, was completed on every day with in-person visits and Hologic software version 4.0 was used for scan analysis. The same DXA scanner was used on all participants, and a single trained research assistant verified all scans and defined body regions for analysis. Whole-body fat percentage, trunk fat mass and trunk to peripheral fat mass ratio were derived from the DXA scan analysis [10]. A phlebotomist performed fasting blood draw in willing participants at early adolescence $(n=579)$. We requested that children were fasting for at least eight hours before the time of the blood draw. Plasma fasting insulin was measured using an electro-chemiluminescence immunoassay on the Roche Modular system and fasting glucose was measured enzymatically using Roche Diagnostics reagents. We estimated insulin resistance using the homeostatic model assessment (HOMA-IR; (glucose in $\mathrm{mg} / \mathrm{dL} \mathrm{x}$ insulin in $\mu \mathrm{U} / \mathrm{mL}$ ) / 405) [11].

\section{Covariates}

At enrollment during the first trimester of pregnancy (median 9.9 weeks), women reported their age, education level, parity, marital status, household income, height, and pre-pregnancy weight, from which we calculated pre-pregnancy BMI $\left(\mathrm{kg} / \mathrm{m}^{2}\right)$. We obtained child's sex from the delivery interview and mothers reported their child's race/ethnicity at the early childhood (3-year) visit.

\section{Statistical analysis}

We categorized characteristics of mothers and children as presented in Table 1 and presented descriptive characteristics for mothers at inclusion and children in early adolescence as mean \pm SD or number (\%). We calculated annual prevalence of each of the four dietary behaviors of interest including all children with at least one early adolescence outcome, and present sex-specific prevalences from ages 4 to 11 ( 4 to 10 for daily breakfast) in Fig. 1. We examined whether the proportions of participants adopting each behavior varied yearly using McNemar's tests. We examined associations of maternal and child characteristics at inclusion with dietary behaviors throughout childhood (age 4 to 11 ) separately in boys and girls using general linear mixed models (PROC GLIMMIX) with a logistic link function (Table 2). We estimated the longitudinal associations of childhood dietary behaviors from age 4 to 11 with adiposity measurements and HOMA-IR in early adolescence using multivariate linear mixed effect models, with the children's age at exposure and covariates as fixed effects, and subject as a random effect (Table 3). The first model was adjusted for age at outcome, while model 2 was additionally adjusted for baseline maternal socio-demographic characteristics as well as child's race/ethnicity. Finally, model 3 was additionally adjusted for maternal pre-pregnancy BMI. We assessed sex interactions in the longitudinal models and we found significant interactions across all behaviors. As a result, we conducted all analyses stratified by sex. Because HOMA-IR was right skewed, we log-transformed this outcome prior to including in the linear mixed model. We also presented associations with HOMA-IR additionally adjusted for current BMI at early adolescence. To simplify the interpretation, we exponentiated regression coefficients and confidence intervals and we presented them as percent change. We conducted all analyses using SAS version 9.4 (SAS Institute).

\section{Results}

Our sample included 504 boys and 491 girls for whom at least one outcome was measured during early adolescence (mean \pm SD: $13.2 \pm 0.9$ years old). Children were mostly white $(65 \%)$ and the majority of mothers had a high education level $(71 \% \geq$ college degree $)$ and 
Table 1 Maternal baseline characteristics and children's characteristics in early adolescence $(N=995)$

\begin{tabular}{|c|c|c|c|}
\hline Maternal characteristics & Overall $(N=995)$ & Boys $(N=504)$ & Girls $(N=491)$ \\
\hline Age, years & $32.4 \pm 5.1$ & $32.2 \pm 5.3$ & $32.6 \pm 4.8$ \\
\hline \multicolumn{4}{|l|}{ Annual household income, US\$ } \\
\hline$>70,000$ & $589(64)$ & $302(66)$ & $287(63)$ \\
\hline$\leq 70,000$ & $327(36)$ & $155(34)$ & $172(37)$ \\
\hline \multicolumn{4}{|l|}{ Maternal education } \\
\hline$\geq$ College degree & $709(71)$ & $348(69)$ & $361(74)$ \\
\hline$<$ College degree & $286(29)$ & $156(31)$ & $130(26)$ \\
\hline \multicolumn{4}{|l|}{ Mother's marital status } \\
\hline Married / cohabitating & $916(92)$ & $466(92)$ & $450(92)$ \\
\hline Other & $79(8)$ & $38(8)$ & $41(8)$ \\
\hline \multicolumn{4}{|l|}{ Parity } \\
\hline Nulliparous & $472(47)$ & $239(47)$ & $233(47)$ \\
\hline$\geq 1$ child & $523(53)$ & $265(53)$ & $258(53)$ \\
\hline Pre-pregnancy BMl, kg/m² & $24.8 \pm 5.2$ & $24.8 \pm 4.9$ & $24.7 \pm 5.5$ \\
\hline \multicolumn{4}{|l|}{ Pre-pregnancy BMI } \\
\hline$\geq 25 \mathrm{~kg} / \mathrm{m}^{2}$ & $367(37)$ & $197(39)$ & $170(35)$ \\
\hline$<25 \mathrm{~kg} / \mathrm{m}^{2}$ & $628(63)$ & $307(61)$ & $321(65)$ \\
\hline Child characteristics & Overall & Boys & Girls \\
\hline \multicolumn{4}{|l|}{ Race / ethnicity } \\
\hline White & $647(65)$ & $315(63)$ & $332(68)$ \\
\hline Black & $29(3)$ & $16(3)$ & $13(3)$ \\
\hline Asian & $157(16)$ & $85(17)$ & $72(15)$ \\
\hline Hispanic & $45(5)$ & $24(5)$ & $21(4)$ \\
\hline Other & $117(12)$ & $64(13)$ & $53(11)$ \\
\hline Age at early adolescence visit, years & $13.2 \pm 0.9$ & $13.2 \pm 1.0$ & $13.2 \pm 0.9$ \\
\hline BMI Z-score, unit ${ }^{b}$ & $0.37 \pm 1.06$ & $0.39 \pm 1.06$ & $0.34 \pm 1.06$ \\
\hline Waist circumference, $\mathrm{cm}$ & $73.0 \pm 11.6$ & $73.3 \pm 12.1$ & $72.8 \pm 11.1$ \\
\hline BI whole-body fat, $\%^{c}$ & $21.8 \pm 10.3$ & $17.5 \pm 9.3$ & $26.2 \pm 9.4$ \\
\hline DXA whole-body fat, $\%^{\mathrm{d}}$ & $28.6 \pm 7.5$ & $26.8 \pm 8.0$ & $30.4 \pm 6.6$ \\
\hline DXA trunk fat, $\mathrm{kg}^{\mathrm{d}}$ & $6.2 \pm 4.0$ & $5.7 \pm 4.0$ & $6.6 \pm 3.9$ \\
\hline Trunk to peripheral fat ratio ${ }^{d}$ & $0.59 \pm 0.12$ & $0.57 \pm 0.11$ & $0.61 \pm 0.12$ \\
\hline Fasting glucose, $\mathrm{mg} / \mathrm{dl}^{\mathrm{e}}$ & $92.9 \pm 23.5$ & $93.2 \pm 18.3$ & $92.6 \pm 28.1$ \\
\hline Fasting insulin, $u U / \mathrm{ml}^{\mathrm{e}}$ & $14.1 \pm 9.0$ & $12.7 \pm 9.0$ & $15.7 \pm 8.9$ \\
\hline HOMA-IR & $3.23 \pm 2.43$ & $2.88 \pm 2.18$ & $3.62 \pm 2.63$ \\
\hline
\end{tabular}

Presented as mean \pm SD or N (\%); BMI Body mass index, BI Bio-impedance, DXA Dual-energy X-ray absorptiometry, HOMA-IR Homeostatic model assessment for insulin resistance

${ }^{\mathrm{a}} N=916 ;{ }^{\mathrm{b}} N=991 ;{ }^{\mathrm{c}} N=982 ;{ }^{\mathrm{d}} N=721 ;{ }^{\mathrm{e}} N=579$

household income (64\%>70,000 \$US), and 92\% were married or cohabitating (Table 1).

The frequency of healthful dietary behaviors either remained stable or decreased slightly with age in both sexes, although not in a linear fashion. Daily breakfast intake remained quite stable from age $4(86 \%)$ to age 10 (84\%; $P=0.12$ ). Daily family dinner decreased, from $57 \%$ at age 4 to $46 \%$ at age $11(P<0.001)$; its decrease mainly started around 9 years old. Fast food consumption less than once per week, decreased slightly, from 77 to $71 \%$ $(P<0.001)$, with a first drop between 6 and 7 years. Finally, eating meals while watching television less than once per week remained stable, 64 and $63 \%$ at ages 4 and $11(P=0.32)$. The proportion of boys eating fast food less than once per week was lower than for girls at 10 (64 vs. $72 \% ; P=0.02$ ) and 11 years old (67 vs. $76 \% ; P=0.01$ ). No other sex-specific differences were observed in frequency of healthful dietary behaviors during childhood. 

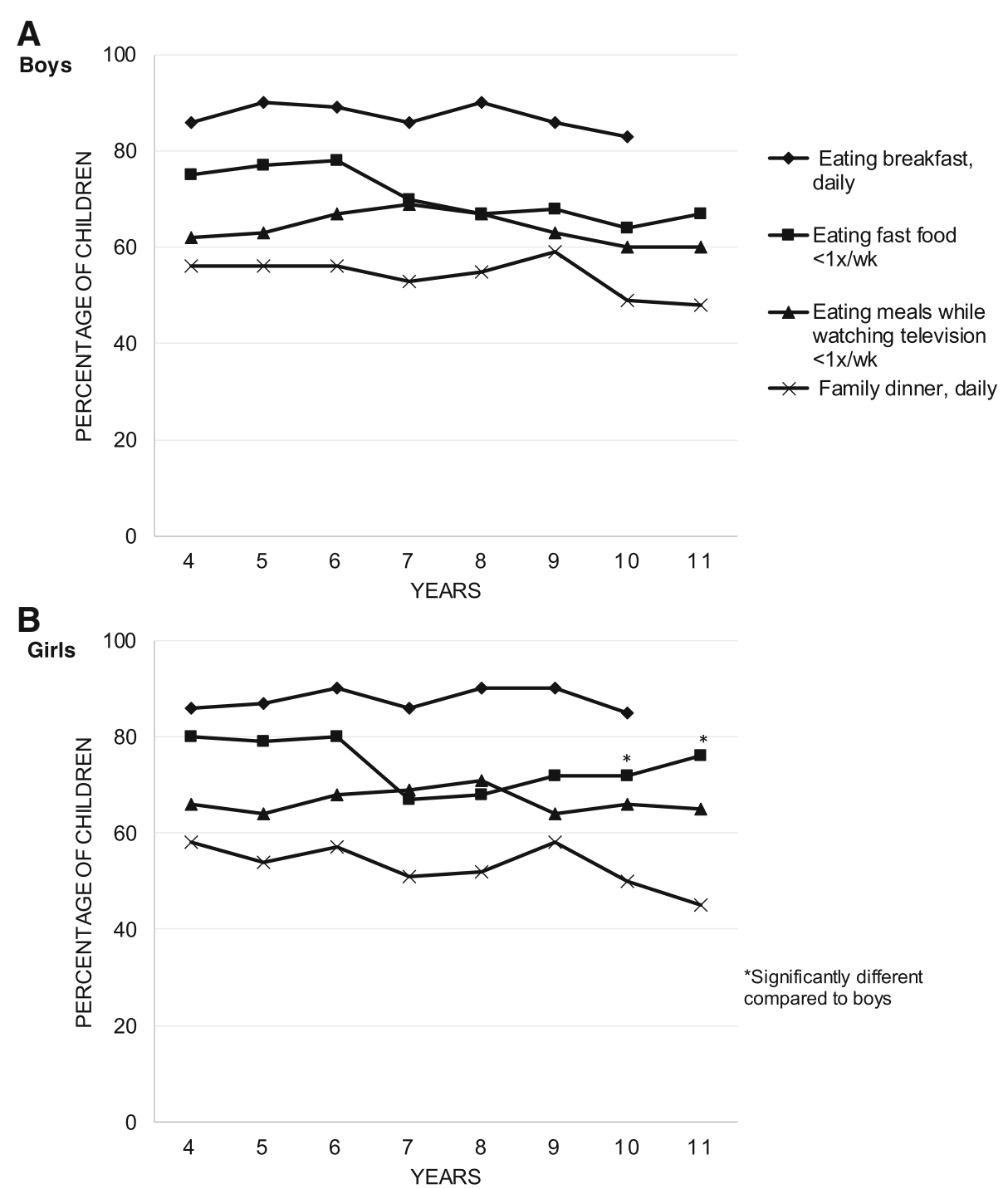

Fig. 1 Trends in dietary behaviors in boys (a) and girls (b) throughout childhood ( $N=297$ to 461 for boys and $N=284$ to 455 for girls)

Both child and maternal characteristics were associated with dietary behaviors in both boys and girls (Table 2). Child's race / ethnicity was associated with all four examined dietary behaviors. A lower household income was associated with lower odds of eating breakfast daily, eating fast food less than once per week and eating meals while watching television less than once per week. A lower maternal education level, not being married or in partnership, and being overweight/obese pre-pregnancy were additionally associated with a lower odds of eating family dinner daily. Parity was not associated with dietary behaviors throughout childhood.

We present sex-stratified adjusted longitudinal associations of dietary behaviors throughout childhood, from age 4 to 11 , with early adolescent (age $13.2 \pm 0.9$ years) adiposity and estimated insulin resistance in Table 3. Overall, the effect estimates for all associations were reduced after adjustment for socio-economic characteristics, and further attenuated after adjustment for maternal pre-pregnancy BMI, although several associations remained significant. In fully adjusted models, eating breakfast daily was associated with lower adiposity measurements (BMI-z, DXA whole-body fat percentage and trunk to peripheral fat ratio) in both boys and girls, as well as with lower HOMA-IR in boys and with lower waist circumference, BI whole-body fat percentage and DXA trunk fat mass in girls. The association with HOMA-IR in boys remained significant after adjustment for current BMI (BMI-adjusted \% difference: - 12.9\%; 95\% CI $[-19.5,-5.7])$. Daily family dinner was associated with lower adiposity (BMI-z, waist circumference, BI whole-body fat percentage, DXA trunk fat mass and trunk to peripheral fat ratio) in girls. In boys, daily family dinner was associated with lower HOMA-IR, an 
Table 2 Associations of maternal and child characteristics with dietary behaviors among Viva children throughout childhood (ages 4 to 11 years; $N=995$ )

\begin{tabular}{|c|c|c|c|c|c|c|c|c|}
\hline & \multicolumn{2}{|c|}{ Eating breakfast daily } & \multicolumn{2}{|c|}{ Eating dinner with family daily } & \multicolumn{2}{|c|}{ Eating fast food $<1 /$ wk } & \multicolumn{2}{|c|}{ Eating meals while watching television $<1 / w k$} \\
\hline & \multicolumn{8}{|l|}{ OR $(95 \% \mathrm{Cl})$} \\
\hline & Boys & Girls & Boys & Girls & Boys & Girls & Boys & Girls \\
\hline \multicolumn{9}{|c|}{ Maternal characteristics } \\
\hline \multicolumn{9}{|c|}{ Household income } \\
\hline $\begin{array}{c}>70,000 \\
\text { US\$ }\end{array}$ & 1.0 (ref) & 1.0 (ref) & 1.0 (ref) & 1.0 (ref) & 1.0 (ref) & 1.0 (ref) & 1.0 (ref) & 1.0 (ref) \\
\hline $\begin{array}{c}\leq 70,000 \\
\text { US\$ }\end{array}$ & $0.55(0.39,0.76)$ & $0.56(0.40,0.79)$ & $0.97(0.77,1.22)$ & $1.04(0.83,1.31)$ & $0.87(0.68,1.11)$ & $0.58(0.45,0.73)$ & $0.76(0.60,0.98)$ & $0.61(0.48,0.78)$ \\
\hline \multicolumn{9}{|c|}{ Maternal education } \\
\hline $\begin{array}{c}\geq \text { College } \\
\text { degree }\end{array}$ & 1.0 (ref) & 1.0 (ref) & 1.0 (ref) & 1.0 (ref) & 1.0 (ref) & 1.0 (ref) & 1.0 (ref) & 1.0 (ref) \\
\hline $\begin{array}{c}<\text { College } \\
\text { degree }\end{array}$ & $0.43(0.31,0.59)$ & $0.45(0.32,0.63)$ & $0.91(0.73,1.15)$ & $0.76(0.59,0.97)$ & $0.61(0.48,0.77)$ & $0.49(0.38,0.64)$ & $0.54(0.42,0.69)$ & $0.46(0.35,0.60)$ \\
\hline \multicolumn{9}{|c|}{ Maternal marital status } \\
\hline $\begin{array}{l}\text { Married / } \\
\text { cohabitating }\end{array}$ & 1.0 (ref) & 1.0 (ref) & 1.0 (ref) & 1.0 (ref) & 1.0 (ref) & 1.0 (ref) & 1.0 (ref) & 1.0 (ref) \\
\hline Other & $0.35(0.21,0.59)$ & $0.39(0.24,0.64)$ & $1.03(0.66,1.61)$ & $0.64(0.42,0.98)$ & $0.91(0.57,1.46)$ & $0.56(0.37,0.85)$ & $0.52(0.33,0.83)$ & $0.45(0.29,0.68)$ \\
\hline \multicolumn{9}{|l|}{ Maternal parity } \\
\hline$\geq 1$ & 1.0 (ref) & 1.0 (ref) & 1.0 (ref) & 1.0 (ref) & 1.0 (ref) & 1.0 (ref) & 1.0 (ref) & 1.0 (ref) \\
\hline Nulliparous & $1.03(0.76,1.41)$ & $1.37(0.99,1.90)$ & $1.02(0.83,1.26)$ & $1.15(0.93,1.43)$ & $1.14(0.91,1.42)$ & $1.24(0.98,1.56)$ & $0.86(0.68,1.07)$ & $1.02(0.81,1.28)$ \\
\hline \multicolumn{9}{|c|}{ Maternal pre-pregnancy BMI } \\
\hline$<25 \mathrm{~kg} / \mathrm{m}^{2}$ & 1.0 (ref) & 1.0 (ref) & 1.0 (ref) & 1.0 (ref) & 1.0 (ref) & 1.0 (ref) & 1.0 (ref) & 1.0 (ref) \\
\hline$\geq 25 \mathrm{~kg} / \mathrm{m}^{2}$ & $0.72(0.53,0.99)$ & $0.65(0.46,0.90)$ & $1.02(0.82,1.26)$ & $0.77(0.61,0.96)$ & $0.81(0.64,1.02)$ & $0.57(0.45,0.73)$ & $0.79(0.63,0.99)$ & $0.69(0.54,0.88)$ \\
\hline \multicolumn{9}{|c|}{ Child characteristics } \\
\hline \multicolumn{9}{|l|}{ Race/ethnicity } \\
\hline White & 1.0 (ref) & 1.0 (ref) & 1.0 (ref) & 1.0 (ref) & 1.0 (ref) & 1.0 (ref) & 1.0 (ref) & 1.0 (ref) \\
\hline Asian & $0.64(0.28,1.49)$ & $0.42(0.19,0.94)$ & $0.84(0.47,1.49)$ & $2.63(1.26,5.50)$ & $0.71(0.39,1.30)$ & $1.53(0.68,3.43)$ & $0.89(0.48,1.67)$ & $2.04(0.86,4.85)$ \\
\hline Black & $0.38(0.26,0.56)$ & $0.35(0.23,0.53)$ & $0.72(0.53,0.97)$ & $0.68(0.49,0.95)$ & $0.50(0.37,0.68)$ & $0.55(0.39,0.77)$ & $0.52(0.38,0.71)$ & $0.38(0.27,0.53)$ \\
\hline Hispanic & $0.48(0.24,0.94)$ & $0.28(0.14,0.55)$ & $1.34(0.79,2.27)$ & $0.99(0.54,1.80)$ & $0.90(0.51,1.59)$ & $0.30(0.17,0.53)$ & $0.95(0.54,1.66)$ & $0.43(0.23,0.79)$ \\
\hline Other & $0.48(0.31,0.75)$ & $1.04(0.58,1.85)$ & $1.04(0.76,1.43)$ & $1.04(0.73,1.47)$ & $0.94(0.66,1.34)$ & $0.80(0.55,1.15)$ & $0.92(0.65,1.30)$ & $0.49(0.34,0.71)$ \\
\hline
\end{tabular}

association that remained significant after adjustment for current BMI (BMI-adjusted \% difference; - 5.5 95\% CI $[-10.2,-0.5])$. Eating fast food less than once per week was associated with lower adiposity (BMI-z, waist circumference and DXA adiposity measurements) in girls as well as with lower HOMA-IR only in boys and girls; however, the associations with HOMA-IR were attenuated after adjustment for current BMI (BMI-adjusted \% difference: $-4.5 \%$; 95\% CI $[-9.7,1.0]$ in boys and $-4.7 \%[-9.9,0.8]$ in girls). Associations of eating meals while watching television with adiposity were mainly seen in boys: eating meals while watching television less than once per week throughout childhood was associated with lower adiposity (BMI-z, waist circumference, BI whole-body fat percentage, and DXA adiposity measurements) and HOMA-IR in boys, an association that was only slightly attenuated when adjusted for current BMI (BMI-adjusted \% difference: - 7.7\%; 95\% CI
$[-12.5,-2.6])$. In girls, eating meals while watching television less than once per week throughout childhood was associated with lower BI whole-body fat percentage.

\section{Discussion}

In this longitudinal cohort, the proportion of children with healthful dietary behaviors decreased slightly from childhood into early adolescence in a non-linear trend, and with only minor differences between girls and boys. Dietary behaviors were associated with child and maternal characteristics as well as socio-economic status. We observed several sex-specific associations between dietary behaviors throughout childhood and adiposity and insulin resistance in early adolescence. Eating breakfast every day was associated with lower adiposity, both overall (BMI-z, whole-body fat percentage) and central (trunk to peripheral fat ratio) in boys and girls, and with lower insulin resistance in boys. Daily family dinner and 
Table 3 Longitudinal associations of dietary behaviors throughout childhood (ages 4 to 11 years) with adiposity and estimated insulin resistance in early adolescence $(N=995)$

\begin{tabular}{|c|c|c|c|c|c|c|}
\hline & \multicolumn{3}{|l|}{ Boys } & \multicolumn{3}{|l|}{ Girls } \\
\hline & \multicolumn{3}{|l|}{$\overline{\beta(95 \% \mathrm{Cl})}$} & \multicolumn{3}{|l|}{$\overline{\beta(95 \% \mathrm{Cl})}$} \\
\hline & Model $1^{\mathrm{a}}$ & Model $2^{b}$ & Model $3^{c}$ & Model $1^{\text {a }}$ & Model $2^{\mathrm{b}}$ & Model $3^{c}$ \\
\hline BMI, Z-Score & $N=502$ & & & $N=489$ & & \\
\hline $\begin{array}{l}\text { Eating breakfast } \\
\text { daily }\end{array}$ & $-0.22(-0.34,-0.11)$ & $-0.18(-0.30,-0.06)$ & $-0.13(-0.24,-0.02)$ & $-0.36(-0.48,-0.24)$ & $-0.19(-0.31,-0.07)$ & $-0.13(-0.23,-0.02)$ \\
\hline $\begin{array}{l}\text { Family dinner } \\
\text { daily }\end{array}$ & $-0.03(-0.11,0.04)$ & $-0.01(-0.09,0.06)$ & $-0.01(-0.09,0.06)$ & $-0.26(-0.33,-0.18)$ & $-0.21(-0.29,-0.14)$ & $-0.17(-0.24,-0.11)$ \\
\hline $\begin{array}{l}\text { Fast food restaurant } \\
<1 / \text { wk }\end{array}$ & $-0.11(-0.19,-0.03)$ & $-0.07(-0.15,0.02)$ & $-0.04(-0.12,0.04)$ & $-0.29(-0.38,-0.21)$ & $-0.19(-0.27,-0.10)$ & $-0.09(-0.17,-0.02)$ \\
\hline $\begin{array}{l}\text { Eating meals while watching } \\
\text { television }<1 / \text { wk }\end{array}$ & $-0.21(-0.29,-0.13)$ & $-0.16(-0.24,-0.08)$ & $-0.13(-0.20,-0.05)$ & $-0.21(-0.29,-0.13)$ & $-0.06(-0.14,0.01)$ & $-0.03(-0.10,0.04)$ \\
\hline Waist circumference, $\mathrm{cm}$ & $N=504$ & & & $N=491$ & & \\
\hline Eating breakfast daily & $-1.29(-2.62,0.03)$ & $-1.11(-2.44,0.23)$ & $-0.60(-1.87,0.68)$ & $-3.98(-5.21,-2.74)$ & $-2.29(-3.48,-1.10)$ & $-1.59(-2.67,-0.51)$ \\
\hline Family dinner daily & $0.06(-0.78,0.91)$ & $0.14(-0.69,0.98)$ & $0.13(-0.67,0.93)$ & $-2.12(-2.89,-1.36)$ & $-1.57(-2.29,-0.84)$ & $-1.14(-1.80,-0.48)$ \\
\hline $\begin{array}{l}\text { Fast food restaurant } \\
<1 / \text { wk }\end{array}$ & $-0.87(-1.80,0.06)$ & $-0.71(-1.64,0.22)$ & $-0.33(-1.22,0.56)$ & $-3.21(-4.07,-2.34)$ & $-2.17(-3.00,-1.35)$ & $-1.23(-1.99,-0.48)$ \\
\hline $\begin{array}{l}\text { Eating meals while } \\
\text { watching television } \\
<1 / \text { wk }\end{array}$ & $-2.14(-3.01,-1.27)$ & $-1.94(-2.82,-1.07)$ & $-1.55(-2.39,-0.71)$ & $-2.44(-3.25,-1.64)$ & $-1.04(-1.81,-0.26)$ & $-0.70(-1.40,0.01)$ \\
\hline BI whole-body fat, \% & $N=499$ & & & $N=483$ & & \\
\hline $\begin{array}{l}\text { Eating breakfast } \\
\text { daily }\end{array}$ & $-1.94(-2.98,-0.90)$ & $-1.38(-2.42,-0.34)$ & $-0.94(-1.92,0.05)$ & $-3.63(-4.69,-2.57)$ & $-1.98(-2.98,-0.97)$ & $-1.47(-2.39,-0.54)$ \\
\hline $\begin{array}{l}\text { Family dinner } \\
\text { daily }\end{array}$ & $-0.08(-0.75,0.58)$ & $0.11(-0.54,0.77)$ & $0.09(-0.53,0.71)$ & $-2.17(-2.83,-1.51)$ & $-1.66(-2.28,-1.04)$ & $-1.34(-1.91,-0.77)$ \\
\hline $\begin{array}{l}\text { Fast food restaurant } \\
<1 / \text { wk }\end{array}$ & $-0.91(-1.64,-0.18)$ & $-0.46(-1.19,0.27)$ & $-0.14(-0.82,0.55)$ & $-2.43(-3.17,-1.68)$ & $-1.39(-2.09,-0.69)$ & $-0.64(-1.29,0.01)$ \\
\hline $\begin{array}{l}\text { Eating meals while watching } \\
\text { television }<1 / \text { wk }\end{array}$ & $-2.09(-2.77,-1.41)$ & $-1.66(-2.35,-0.98)$ & $-1.33(-1.98,-0.69)$ & $-2.32(-3.01,-1.62)$ & $-0.98(-1.64,-0.32)$ & $-0.74(-1.35,-0.14)$ \\
\hline DXA whole-body fat, \% & $N=355$ & & & $N=366$ & & \\
\hline Eating breakfast daily & $-1.78(-2.80,-0.76)$ & $-1.75(-2.78,-0.72)$ & $-1.43(-2.42,-0.45)$ & $-2.34(-3.21,-1.47)$ & $-1.93(-2.77,-1.09)$ & $-1.47(-2.25,-0.68)$ \\
\hline Family dinner daily & $-0.10(-0.77,0.57)$ & $-0.04(-0.71,0.63)$ & $-0.16(-0.80,0.48)$ & $-0.59(-1.14,-0.04)$ & $-0.49(-1.02,0.03)$ & $-0.29(-0.78,0.19)$ \\
\hline Fast food restaurant $<1 / w k$ & $-0.07(-0.79,0.65)$ & $-0.01(-0.73,0.72)$ & $0.21(-0.48,0.90)$ & $-1.78(-2.40,-1.15)$ & $-1.28(-1.88,-0.68)$ & $-0.89(-1.45,-0.33)$ \\
\hline $\begin{array}{l}\text { Eating meals while } \\
\text { watching television }<1 / \text { wk }\end{array}$ & $-1.30(-1.99,-0.62)$ & $-1.25(-1.94,-0.55)$ & $-1.10(-1.77,-0.44)$ & $-0.88(-1.46,-0.30)$ & $-0.39(-0.95,0.17)$ & $-0.15(-0.67,0.37)$ \\
\hline DXA trunk fat mass, $\mathrm{kg}$ & $N=355$ & & & $N=366$ & & \\
\hline Eating breakfast daily & $-0.65(-1.16,-0.15)$ & $-0.57(-1.08,-0.06)$ & $-0.40(-0.88,0.08)$ & $-1.78(-2.26,-1.29)$ & $-1.23(-1.69,-0.78)$ & $-0.92(-1.33,-0.51)$ \\
\hline Family dinner daily & $0.13(-0.20,0.46)$ & $0.16(-0.16,0.49)$ & $0.10(-0.21,0.41)$ & $-0.60(-0.91,-0.30)$ & $-0.45(-0.74,-0.16)$ & $-0.32(-0.57,-0.06)$ \\
\hline Fast food restaurant $<1 / w k$ & $-0.10(-0.45,0.26)$ & $-0.01(-0.36,0.35)$ & $0.11(-0.22,0.44)$ & $-1.29(-1.64,-0.94)$ & $-0.86(-1.19,-0.53)$ & $-0.60(-0.90,-0.31)$ \\
\hline $\begin{array}{l}\text { Eating meals while } \\
\text { watching television }<1 / \text { wk }\end{array}$ & $-0.71(-1.05,-0.38)$ & $-0.63(-0.97,-0.29)$ & $-0.56(-0.88,-0.23)$ & $-0.74(-1.06,-0.42)$ & $-0.31(-0.61,0.00)$ & $-0.15(-0.42,0.12)$ \\
\hline Trunk to peripheral fat ratio & $N=355$ & & & $N=366$ & & \\
\hline Eating breakfast daily & $-0.02(-0.04,-0.01)$ & $-0.02(-0.04,-0.01)$ & $-0.02(-0.03,-0.01)$ & $-0.06(-0.08,-0.05)$ & $-0.06(-0.07,-0.04)$ & $-0.05(-0.06,-0.03)$ \\
\hline Family dinner daily & $0.01(0.00,0.02)$ & $0.01(0.00,0.02)$ & $0.01(0.00,0.02)$ & $-0.02(-0.03,-0.01)$ & $-0.02(-0.03,-0.01)$ & $-0.02(-0.03,-0.01)$ \\
\hline Fast food restaurant $<1 / w k$ & $-0.01(-0.02,0.00)$ & $-0.01(-0.02,0.00)$ & $0.00(-0.01,0.00)$ & $-0.04(-0.05,-0.03)$ & $-0.03(-0.04,-0.02)$ & $-0.03(-0.04,-0.02)$ \\
\hline $\begin{array}{l}\text { Eating meals while } \\
\text { watching television }<1 / \text { wk }\end{array}$ & $-0.02(-0.03,-0.01)$ & $-0.02(-0.03,-0.01)$ & $-0.02(-0.03,-0.01)$ & $-0.03(-0.04,-0.02)$ & $-0.02(-0.03,-0.01)$ & $-0.01(-0.02,0.00)$ \\
\hline
\end{tabular}


Table 3 Longitudinal associations of dietary behaviors throughout childhood (ages 4 to 11 years) with adiposity and estimated insulin resistance in early adolescence $(N=995)$ (Continued)

\begin{tabular}{|c|c|c|c|c|c|c|}
\hline & \multicolumn{3}{|l|}{ Boys } & \multicolumn{3}{|l|}{ Girls } \\
\hline & \multicolumn{3}{|l|}{$\beta(95 \% \mathrm{Cl})$} & \multicolumn{3}{|l|}{$\beta(95 \% \mathrm{Cl})$} \\
\hline & Model $1^{a}$ & Model $2^{b}$ & Model $3^{c}$ & $\overline{\text { Model } 1^{\text {a }}}$ & Model $2^{\mathrm{b}}$ & Model $3^{c}$ \\
\hline & $\%$ difference $(95 \% \mathrm{Cl})$ & & & $\%$ difference $(95 \% \mathrm{Cl})$ & & \\
\hline HOMA-IR & $N=304$ & & & $N=275$ & & \\
\hline Eating breakfast daily & $-20.1(-26.8,-12.9)$ & $-17.7(-24.7,-10.1)$ & $-15.6(-22.7,-7.9)$ & $-17.1(-23.8,-9.7)$ & $-9.2(-16.5,-1.2)$ & $-7.8(-15.1,0.1)$ \\
\hline Family dinner daily & $-8.0(-13.2,-2.6)$ & $-6.8(-12.0,-1.3)$ & $-7.3(-12.4,-1.8)$ & $-7.0(-12.0,-1.7)$ & $-3.1(-8.2,2.2)$ & $-3.2(-8.1,2.1)$ \\
\hline Fast food restaurant $<1 / \mathrm{wk}$ & $-10.1(-15.5,-4.3)$ & $-8.3(-13.9,-2.4)$ & $-7.6(-13.2,-1.7)$ & $-13.3(-18.5,-7.7)$ & $-8.5(-13.8,-2.8)$ & $-6.6(-12.0,-0.9)$ \\
\hline $\begin{array}{l}\text { Eating meals while } \\
\text { watching television }<1 / \text { wk }\end{array}$ & $-13.9(-18.9,-8.6)$ & $-11.7(-16.8,-6.2)$ & $-10.7(-15.8,-5.2)$ & $-3.4(-8.8,2.3)$ & $3.1(-2.5,9.1)$ & $3.7(-1.8,9.6)$ \\
\hline
\end{tabular}

BMI Body mass index, HOMA-IR Homeostatic model assessment for insulin resistance, $B I$ Bio-impedance, DXA Dual-energy X-ray absorptiometry. ${ }^{\mathrm{a} A d j u s t e d}$ for child's age at exposures and outcome (except for BMI Z-Score); ${ }^{b}$ Model 1 additionally adjusted for child race/ethnicity and maternal education, marital status, and parity, 'Model 2 additionally adjusted for pre-pregnancy BMI

eating fast food less than once per week were both associated with lower adiposity in girls and lower insulin resistance in boys. Finally, eating meals while watching television was associated with adiposity and insulin resistance mainly in boys.

Our results are mostly in line with previously published observational reports for trends in dietary behaviors during childhood, but add to current literature with our longitudinal assessments. Breakfast frequency in children and adolescents was investigated in several studies in various populations, and these studies consistently showed a decrease in breakfast consumption from childhood to adolescence [12-15]. Important variations in breakfast eating across countries and regions have been reported, and lower odds of eating breakfast daily were observed in girls, children from a family with a lower socio-economic status and a family structure that did not include two parents [12]. Throughout childhood, we found an even proportion of children eating breakfast daily between ages four and ten; however, we found an association of daily breakfast eating with maternal household income, education and marital status, as well as with maternal BMI and child's race/ethnicity, but not with child's sex. Observational and longitudinal studies in children have suggested that regular breakfast consumption plays a role in the prevention of excess adiposity and obesity during childhood and adolescence [15], despite higher energy intakes with regular breakfast consumption [13]. The overall nutritional profile is typically improved with regular breakfast eating, including higher intakes of micronutrients, fruits, whole grains and dairy [16]. Eating breakfast could also impact global dietary quality and energy intakes beyond breakfast itself by influencing hunger and satiety for the remaining meals [15]. However, results from intervention studies including breakfast initiation as a weight loss strategy have yielded inconclusive results [17] and a recent review suggested positive to neutral support for the inclusion of breakfast for improvements in appetite control, satiety, and postprandial energy expenditure [18]. In the present study, we showed that daily breakfast consumption throughout childhood had the strongest association of all behaviors examined with lower adiposity in early adolescence in both boys and girls, and we added to the previous studies by demonstrating an association with lower insulin resistance in boys. Our findings thus corroborate results from previous observational studies, and our longitudinal assessment suggest that long-term breakfast consumption, starting at an earlier age could be a key element explaining some of the breakfast benefits. Our results also suggest that amongst childhood dietary behaviors, breakfast habits could possibly be one of the most important for both boys and girls on which to focus interventions.

The benefits of family dinner have also been studied quite extensively. For example, in the Growing Up Today Study (GUTS), $50.7 \%$ of children ate dinner together with family every day at age 9 , a proportion that decreased to $35.4 \%$ by age 14 [19]. Although the proportion of children eating dinner with their family daily was slightly higher in our study sample at age 9 (58\%), a similar decrease was observed with $46 \%$ of children eating dinner with their family daily by age 11 . Eating meals together with family may contribute to healthful dietary intakes such as increased fruit and vegetables consumption and less fried food and soda [19-21]. In addition, family dinner frequency has been associated with childhood obesity in some [19, 21], but not all studies [22]. In GUTS, the frequency of eating dinner together with family was not associated with the likelihood of becoming overweight in longitudinal analyses and no sex-specific differences were reported [22]. In contrast, we found an association between daily family dinner throughout childhood and BMI-z as well as some adiposity measurements in girls in early adolescence. This discrepancy could be explained by the children's age at assessment. In GUTS, children were 9 to 14 years of age at inclusion while we included children starting at 4 
years old, thus suggesting greater effects of this behavior if sustained starting at younger ages.

Fast food consumption has on average increased over the past decades and increases with age across childhood and adolescence [23, 24]. In the 2011-2012 National Health and Nutrition Examination Survey (NHANES), a lower caloric intake from fast food was found in non-Hispanic Asian children and no differences were noted by sex and poverty status [24]. Fast food meals and food items are typically energy-dense and have a poor nutrient profile. Fast food consumption in children has been associated with higher energy, fat, sodium, and sugar intakes as well as lower intakes of fruits, vegetables and fiber $[25,26]$. Results from cross-sectional studies in children investigating the association between higher fast food intake and BMI are somewhat inconsistent [27], but results from GUTS (9 to 14 years old) showed that a yearly increase (from never or $<$ once per week to 4-7 times per week) in the consumption of fried food away from home was associated with higher BMI compared to children with a constant low intake of fried food away from home, with similar findings between girls and boys [28]. However, we found associations of fast food consumption throughout childhood with adiposity only in girls and with insulin resistance in boys and girls. Mechanisms including caloric intake compensation or responsiveness to internal cues of hunger and satiety should be investigated to try and explain these differences between boys and girls.

To our knowledge, no study has reported trends in eating meals while watching television across childhood or associations of eating while watching television with adolescence obesity or adiposity. Watching television itself has been associated with obesity, in part due to increased sedentary time, but it could also be related to dietary behaviors and choices $[29,30]$. Moreover, eating meals or snacks while watching television is common in children and it has been associated with increased energy intakes, poorer diet quality and increased intakes of sugar and fat, snack food, and sugar-sweetened beverages, as well as lower intakes of fruits and vegetables $[31,32]$. These effects of eating while watching television could be explained by the increased exposure to unhealthy food advertisement targeting children [33] or to the effects of "mindless or inattentive eating" [34]. Again, we observed sex-specific associations for this behavior, with eating meals while watching television being associated with adiposity and insulin resistance mainly in boys. Future studies should examine underlying mechanisms explaining these sex-related differences.

A key finding of our study was that several healthful dietary behaviors throughout childhood were associated with lower estimated insulin resistance in boys in early adolescence, some independently of BMI. Type 2 diabetes is a growing health concern in children [35]. Unhealthy lifestyle behaviors during childhood combined with increased body weight likely contribute to the risk of prediabetes; however, the contributing role of dietary behaviors throughout childhood remains scarcely studied. Our results indicate that daily breakfast eating, daily family dinner, and eating meals while watching television less than once per week throughout childhood were associated with lower early adolescence HOMA-IR in boys, even after adjustment for current BMI-z, suggesting potential benefits of these behaviors beyond weight control. Our results support the need for nutrition education for diabetes prevention in youth.

Our findings also highlight the importance of adopting healthful dietary behaviors early on, and maintaining them throughout childhood. Other studies have shown that behaviors set in childhood are likely to become habits and carry into adulthood, further increasing the long-course benefits of early healthful behaviors [36-38]. For example, the longitudinal association between diet quality and lifestyle behaviors was examined in the NEXT Plus Study [36]. Over a 4-year period from adolescence to adulthood, breakfast, family meals and eating meals while watching television decreased while fast food consumption increased. Breakfast and family meals as well as less frequent fast food and meals during television viewing throughout late adolescence were all associated with healthier diet quality in young adulthood [36].

An expert committee including representatives of 15 national health care organizations published recommendations regarding the prevention, assessment and treatment of child and adolescent overweight and obesity [39]. Their recommendations included a list of healthful lifestyle habits recognized as effective in preventing excessive weight gain. The following behaviors were included in this list: eating breakfast daily, limiting eating out at restaurants, particularly fast food restaurants, and encouraging family meals in which parents and children eat together [39]. Avoiding eating while watching television was not included in the list, although limiting screen and television time was. Our results provide empiric evidence to support the importance of these dietary behaviors starting early and maintained throughout childhood and their potential significance for family-based interventions targeting childhood obesity. Moreover, we have identified several associations between socio-demographic characteristics (household income, maternal education, marital status, and pre-pregnancy BMI, and child's race ethnicity) and dietary behaviors, highlighting the need to consider socio-economic status in the development of dietary interventions. In addition, several other lifestyle behaviors including, for example, physical activity, sleep patterns and sedentary behaviors or screen time have been associated 
with child's adiposity [39-43]. Although not included in the present analysis, these lifestyle behaviors could be potential mediators of the observed associations and should be considered for future family-based interventions.

Strengths of this study include its large sample size and the longitudinal data collected prospectively over nearly ten years. Very few prior studies have investigated dietary behaviors throughout childhood including multiple time points to investigate their long-term effects through adolescence. To our knowledge, this is also the first study examining longitudinal associations of dietary behaviors and insulin resistance in adolescents. Whether these dietary behaviors remain associated with adiposity or insulin resistance and prediabetes risk during the transition from adolescence to adulthood should be investigated. Our analyses were adjusted for several maternal and child socio-demographic characteristics; however, our study sample was mainly white, with a higher family income and education level, therefore limiting the generalizability of the results. Another limitation is that we collected self-reported pre-pregnancy weight at the initial prenatal visit, and maternal BMI was a strong confounder of the relationship between child behaviors and BMI. However, among 343 women who had weight recorded in the medical record in the 3 months before their last menstrual period, the association between self-reported and clinically measured weight was linear $(r=0.997)$ [44]. Also, dietary behaviors were assessed by mothers from age 4 to 8 , and by children from age 9 to 11 , and we cannot exclude that maternal or child answers may differ; yet, whether one or the other is more reliable is unknown. At age 9, dietary behaviors were assessed in both maternal and child questionnaires, and accordance between answers was modest (kappa coefficients $=0.18$ for eating while watching television less than once per week, 0.48 for eating breakfast daily, 0.18 for eating fast food less than once per week and 0.42 for eating dinner with family daily). In addition, insulin resistance was estimated from fasting insulin and glucose levels and not with gold-standard clamps; yet, HOMA-IR has been shown to have a high sensitivity and specificity for dynamic indices of insulin resistance measured during an oral glucose tolerance test in obese adolescents [45]. Finally, we could not assess whether these healthful dietary behaviors were associated with adiposity and body weight through reduced caloric intake or improved dietary quality, or through different mechanisms.

\section{Conclusions}

In conclusion, the frequency of healthful dietary behaviors decreases slightly in childhood with advancing age. Healthful dietary behaviors throughout childhood are associated with lower adiposity measures as well as lower estimated insulin resistance in early adolescence, and sex-specific associations were identified. Daily breakfast eating appeared to have the largest effect and most consistent association amongst the examined behaviors in both boys and girls. Associations of fast food consumption with adiposity were mainly observed in girls, and associations of eating while watching television with adiposity and insulin resistance were seen mostly in boys. Healthier dietary behaviors, which are possibly associated with a healthier overall diet quality, could be translated into simple and applicable dietary advice for families and communities. The effectiveness of such interventions in various socio-demographic settings remains to be assessed.

\section{Additional file}

Additional file 1: Figure S1. Flow-chart of participants included in the longitudinal analysis. Figure S2. Proportion of children included in the analysis ( $N=995)$ with available questionnaires each year. (DOCX $31 \mathrm{~kb}$ )

\section{Abbreviations}

BI: Bio-impedance; BMI: Body mass index; DXA: Dual-energy X-ray absorptiometry; GUTS: Growing Up Today Study; HOMA-IR: Homeostatic model assessment for insulin resistance; NHANES: National Health and Nutrition Examination Survey; WC: Waist circumference

\section{Acknowledgements \\ We thank the participants and staff of Project Viva.}

\section{Funding}

This research is supported by grants from the US National Institutes of Health (R01 HD 034568, UG3OD023286). The content is solely the responsibility of the authors. VG is supported by a Postdoctoral Fellowship from the Department of Population Medicine of Harvard Pilgrim Health Care Institute and Harvard Medical School (Pyle Fellowship).

\section{Availability of data and materials}

The datasets analysed during the current study are not publicly available because we have not obtained consent for public data sharing from all participants but are available from the corresponding author on reasonable request.

\section{Authors' contributions}

VG and MFH: designed the analysis; VG analyzed and interpreted the data, and drafted the manuscript; SRS: provided guidance on statistical analysis; SRS, ET, EO and MFH: provided critical intellectual contributions and read and approved the final manuscript.

\section{Ethics approval and consent to participate}

The institutional review board of Harvard Pilgrim Health Care approved this study and all procedures were in accordance with the ethical standards for human experimentation established by the Declaration of Helsinki. All women provided written informed consent at enrollment and each postnatal follow-up visit and children provided assent at the early adolescent visit.

\section{Consent for publication}

Not applicable.

\section{Competing interests}

The authors declare that they have no competing interests.

\section{Publisher's Note}

Springer Nature remains neutral with regard to jurisdictional claims in published maps and institutional affiliations. 


\section{Author details}

'Division of Chronic Disease Research Across the Lifecourse, Department of Population Medicine, Harvard Medical School and Harvard Pilgrim Health Care Institute, Landmark Center, 401 Park Drive Suite 401, Boston, MA 02215, USA. ${ }^{2}$ Division of General Academic Pediatrics, Department of Pediatrics, Massachusetts General Hospital for Children, Boston, MA, USA. ${ }^{3}$ Department of Nutrition, Harvard T.H. Chan School of Public Health, Boston, MA, USA. ${ }^{4}$ Diabetes Unit, Massachusetts General Hospital, Boston, MA, USA.

Received: 15 May 2018 Accepted: 3 December 2018

\section{Published online: 17 December 2018}

\section{References}

1. Wang Y, Lobstein T. Worldwide trends in childhood overweight and obesity. Int J Pediatr Obes. 2006;1(1):11-25.

2. Nadeau K, Dabelea D. Epidemiology of type 2 diabetes in children and adolescents. Endocr Res. 2008;33(1-2):35-58.

3. Mayer-Davis EJ, Lawrence JM, Dabelea D, Divers J, Isom S, Dolan L, et al. Incidence trends of type 1 and type 2 diabetes among youths, 2002-2012. N Engl J Med. 2017;376(15):1419-29.

4. Sahoo K, Sahoo B, Choudhury AK, Sofi NY, Kumar R, Bhadoria AS. Childhood obesity: causes and consequences. J Family Med Prim Care. 2015;4(2):187-92.

5. Karnik S, Kanekar A. Childhood obesity: a global public health crisis. Int J Prev Med. 2012;3(1):1-7.

6. Patrick $H$, Nicklas TA. A review of family and social determinants of children's eating patterns and diet quality. J Am Coll Nutr. 2005;24(2):83-92.

7. Oken E, Baccarelli AA, Gold DR, Kleinman KP, Litonjua AA, De Meo D, et al. Cohort profile: project viva. Int J Epidemiol. 2015;44(1):37-48.

8. Kuczmarski RJ, Ogden CL, Guo SS, Grummer-Strawn LM, Flegal KM, Mei Z, et al. CDC growth charts for the United States: methods and development. Vital Health Stat 11. 2000;2002(246):1-190.

9. Centers for Disease Control and Prevention. NHANES Anthropometry Procedures Manual. 2000: p 3-30 and 33-31.

10. Vanltallie TB, Yang MU, Heymsfield SB, Funk RC, Boileau RA. Heightnormalized indices of the body's fat-free mass and fat mass: potentially useful indicators of nutritional status. Am J Clin Nutr. 1990;52(6):953-9.

11. Matthews DR, Hosker JP, Rudenski AS, Naylor BA, Treacher DF, Turner RC. Homeostasis model assessment: insulin resistance and beta-cell function from fasting plasma glucose and insulin concentrations in man. Diabetologia. 1985;28(7):412-9.

12. Vereecken C, Dupuy M, Rasmussen M, Kelly C, Nansel TR, Al Sabbah H, et al. Breakfast consumption and its socio-demographic and lifestyle correlates in schoolchildren in 41 countries participating in the HBSC study. Int J Public Health. 2009;54(Suppl 2):180-90.

13. Rampersaud GC, Pereira MA, Girard BL, Adams J, Metzl JD. Breakfast habits, nutritional status, body weight, and academic performance in children and adolescents. J Am Diet Assoc. 2005;105(5):743-60 quiz 761-742.

14. Deshmukh-Taskar PR, Nicklas TA, O'Neil CE, Keast DR, Radcliffe JD, Cho S. The relationship of breakfast skipping and type of breakfast consumption with nutrient intake and weight status in children and adolescents: the National Health and nutrition examination survey 1999-2006. J Am Diet Assoc. 2010;110(6):869-78.

15. Blondin SA, Anzman-Frasca S, Djang HC, Economos CD. Breakfast consumption and adiposity among children and adolescents: an updated review of the literature. Pediatr Obes. 2016;11(5):333-48.

16. Ramsay SA, Bloch TD, Marriage B, Shriver LH, Spees CK, Taylor CA. Skipping breakfast is associated with lower diet quality in young US children. Eur J Clin Nutr. 2018; 72(4):548-56.

17. Dhurandhar EJ, Dawson J, Alcorn A, Larsen LH, Thomas EA, Cardel M, et al. The effectiveness of breakfast recommendations on weight loss: a randomized controlled trial. Am J Clin Nutr. 2014;100(2):507-13.

18. Gwin JA, Leidy HJ. A review of the evidence surrounding the effects of breakfast consumption on mechanisms of weight management. Adv Nutr. 2018; 9(6):717-25.

19. Gillman MW, Rifas-Shiman SL, Frazier AL, Rockett HR, Camargo CA Jr, Field $A E$, et al. Family dinner and diet quality among older children and adolescents. Arch Fam Med. 2000;9(3):235-40.

20. Larson NI, Neumark-Sztainer D, Hannan PJ, Story M. Family meals during adolescence are associated with higher diet quality and healthful meal patterns during young adulthood. J Am Diet Assoc. 2007;107(9):1502-10.
21. Gable S, Chang Y, Krull JL. Television watching and frequency of family meals are predictive of overweight onset and persistence in a national sample of school-aged children. J Am Diet Assoc. 2007;107(1):53-61.

22. Taveras EM, Rifas-Shiman SL, Berkey CS, Rockett HR, Field AE, Frazier AL, et al. Family dinner and adolescent overweight. Obes Res. 2005;13(5):900-6.

23. Niemeier HM, Raynor HA, Lloyd-Richardson EE, Rogers ML, Wing RR. Fast food consumption and breakfast skipping: predictors of weight gain from adolescence to adulthood in a nationally representative sample. J Adolesc Health. 2006;39(6):842-9.

24. Vikraman $S$, Fryar CD, Ogden CL. Caloric intake from fast food among children and adolescents in the United States. NCHS Data Brief. 2011-2012; 2015(213):1-8.

25. Bowman SA, Gortmaker SL, Ebbeling CB, Pereira MA, Ludwig DS. Effects of fast-food consumption on energy intake and diet quality among children in a national household survey. Pediatrics. 2004;113(1 Pt 1):112-8.

26. Paeratakul S, Ferdinand DP, Champagne CM, Ryan DH, Bray GA. Fast-food consumption among US adults and children: dietary and nutrient intake profile. J Am Diet Assoc. 2003;103(10):1332-8.

27. Rosenheck R. Fast food consumption and increased caloric intake: a systematic review of a trajectory towards weight gain and obesity risk. Obes Rev. 2008;9(6):535-47.

28. Taveras EM, Berkey CS, Rifas-Shiman SL, Ludwig DS, Rockett HR, Field AE, et al. Association of consumption of fried food away from home with body mass index and diet quality in older children and adolescents. Pediatrics. 2005;116(4):e518-24.

29. Miller SA, Taveras EM, Rifas-Shiman SL, Gillman MW. Association between television viewing and poor diet quality in young children. Int J Pediatr Obes. 2008;3(3):168-76.

30. Ghobadi S, Hassanzadeh-Rostami Z, Salehi-Marzijarani M, Bellissimo N, Brett NR, Totosy de Zepetnek JO, et al. Association of eating while television viewing and overweight/obesity among children and adolescents: a systematic review and meta-analysis of observational studies. Obes Rev. 2018;19(3):313-20

31. Liang T, Kuhle S, Veugelers PJ. Nutrition and body weights of Canadian children watching television and eating while watching television. Public Health Nutr. 2009;12(12):2457-63.

32. Avery A, Anderson C, McCullough F. Associations between children's diet quality and watching television during meal or snack consumption: a systematic review. Matern Child Nutr. 2017;13(4): e12428.

33. Harrison K, Marske AL. Nutritional content of foods advertised during the television programs children watch most. Am J Public Health. 2005;95(9): 1568-74.

34. Robinson E, Aveyard P, Daley A, Jolly K, Lewis A, Lycett D, et al. Eating attentively: a systematic review and meta-analysis of the effect of food intake memory and awareness on eating. Am J Clin Nutr. 2013;97(4):728-42.

35. Writing Group for the SEARCH for Diabetes in Youth Study Group, Dabelea D, Bell RA, D'Agostino RB Jr, Imperatore G, Johansen JM, Linder B, Liu LL, Loots $B$, Marcovina $S$, et al. Incidence of diabetes in youth in the United States. JAMA. 2007;297(24):2716-24.

36. Lipsky LM, Nansel TR, Haynie DL, Liu D, Li K, Pratt CA, et al. Diet quality of US adolescents during the transition to adulthood: changes and predictors. Am J Clin Nutr. 2017;105(6):1424-32.

37. Mikkila V, Rasanen L, Raitakari OT, Pietinen P, Viikari J. Longitudinal changes in diet from childhood into adulthood with respect to risk of cardiovascular diseases: the cardiovascular risk in young Finns study. Eur J Clin Nutr. 2004; 58(7):1038-45

38. Craigie AM, Lake AA, Kelly SA, Adamson AJ, Mathers JC. Tracking of obesityrelated behaviours from childhood to adulthood: a systematic review. Maturitas. 2011;70(3):266-84.

39. Barlow SE, Expert C. Expert committee recommendations regarding the prevention, assessment, and treatment of child and adolescent overweight and obesity: summary report. Pediatrics. 2007;120(Suppl 4):S164-92.

40. Collings PJ, Westgate K, Vaisto J, Wijndaele K, Atkin AJ, Haapala EA, et al. Cross-sectional associations of objectively-measured physical activity and sedentary time with body composition and cardiorespiratory fitness in midchildhood: the PANIC study. Sports Med. 2017;47(4):769-80.

41. Taveras EM, Gillman MW, Pena MM, Redline S, Rifas-Shiman SL. Chronic sleep curtailment and adiposity. Pediatrics. 2014;133(6):1013-22.

42. Mitchell JA, Dowda M, Pate RR, Kordas K, Froberg K, Sardinha LB, et al. Physical activity and pediatric obesity: a quantile regression analysis. Med Sci Sports Exerc. 2017;49(3):466-73. 
43. Katzmarzyk PT, Barreira TV, Broyles ST, Champagne CM, Chaput JP, Fogelholm M, et al. Relationship between lifestyle behaviors and obesity in children ages 9-11: results from a 12-country study. Obesity (Silver Spring). 2015;23(8):1696-702.

44. Provenzano AM, Rifas-Shiman SL, Herring SJ, Rich-Edwards JW, Oken E. Associations of maternal material hardships during childhood and adulthood with prepregnancy weight, gestational weight gain, and postpartum weight retention. J Women's Health (Larchmt). 2015;24(7):563-71.

45. Keskin M, Kurtoglu S, Kendirci M, Atabek ME, Yazici C. Homeostasis model assessment is more reliable than the fasting glucose/insulin ratio and quantitative insulin sensitivity check index for assessing insulin resistance among obese children and adolescents. Pediatrics. 2005;115(4):e500-3.

Ready to submit your research? Choose BMC and benefit from:

- fast, convenient online submission

- thorough peer review by experienced researchers in your field

- rapid publication on acceptance

- support for research data, including large and complex data types

- gold Open Access which fosters wider collaboration and increased citations

- maximum visibility for your research: over $100 \mathrm{M}$ website views per year

At BMC, research is always in progress.

Learn more biomedcentral.com/submissions 